\title{
Unparticle Searches Through Low Energy Parity Violating Asymmetry
}

\author{
K. O. Ozansoy ${ }^{*}$ \\ Department of Physics, Ankara University, 06100 Tandogan, Ankara, Turkey
}

(Dated: November 3, 2018)

\begin{abstract}
In this paper, we study the effects of the unparticles on the parity-violating asymmetry for the low energy electron-electron scattering, $e^{-} e^{-} \rightarrow e^{-} e^{-}$. Using the data from the E158 experiment at SLAC we extract the limits on the unparticle coupling $\lambda_{A V}$, and on the the energy scale $\Lambda$ at 95\% C.L. for various values of the scaling dimension $d$.
\end{abstract}

\section{INTRODUCTION}

The Standard Model(SM) of electroweak interactions has been throughly a paradigm of the elementary particle physics for more than three decades. It has lived through with the many sucessful predictions and explanations on the experimental observations. Many of its predictions has been tested sucessfully at the high energy collider experiments as well as the low energy experiments. It seems next decade will be the LHC era for testing the SM predictions and searching new physics signatures from beyond the SM. High energy experiments are crucial for direct observation of the signatures from the new physics effects, however, low energy particle physics experiments are also very important to understand the effects in detail.

Low energy parity-violation(PV) measurments are very sensitive to new physics effects around TeV energies, and are complementary to high energy colliders. A comprehensive study on the new physics searches through the low energy parity-violation experiments has been given in Ref. [1]. Atomic PV experiments, and electron-hadron, and electronelectron scattering PV experiments have been performed with very high sensitivity. Amidst those experiments, PV Möller scattering, which is the cleanest since it is a pure leptonic process, is very promising to search for the new physics. There are many works on the low energy parity violating Möller scattering in the literature. For example,

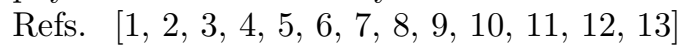

One of the most promising high precision PV measurmment in the Möller scattering was designed by the E158 experiment at the Sanford Linear Accelerator Laboratory(SLAC) (see, for example Refs. [4, 5, 9, 10, 11]). In the experiment, the PV asymmetry measurment is obtained in the scattering of longitudinally polarized electron beams on an unpolarized fixed target. The parity-violating, left-right, scattering asymmetry is defined by

$$
A_{P V}=\frac{\sigma_{R}-\sigma_{L}}{\sigma_{R}+\sigma_{L}}
$$

where $\sigma_{R(L)}$ is the scattering cross section for incedent right(left)-handed electrons.

The high precision measurement of the PV asymmetry $A_{P V}$ has been considered to search for the new physics effects. New physics effects due to $Z^{\prime}$ bosons, compositeness, anomalous anapole moment effects, double charged scalar bosons, extra dimensions, etc. have been considered regarding this parity violating asymmetry measurements, Ref.s [1, 2, 3].

Recently, Georgi has proposed a mind-bending offspring of a possible scale invariant sector living at very high energy scale, Ref [14]. According to this new physics proposal, if there is a conformal symmetry in nature it must be broken at a very high energy scale which is above the current energy scale of the colliders. Considering the idea of the Ref. [15], in the Ref. [14], the scale invariant sector is presented by a set of the Banks-Zaks operators $\mathcal{O}_{B Z}$, and defined at the very high energy scale. Interactions of the BZ operators $\mathcal{O}_{B Z}$ with the SM operators $\mathcal{O}_{S M}$ are expressed by the exchange of particles with a very high energy mass scale $\mathcal{M}_{\mathcal{U}}^{k}$ in the following form

$$
\frac{1}{\mathcal{M}_{\mathcal{U}}^{k}} O_{B Z} O_{S M}
$$

where BZ, and SM operators are defined as $O_{B Z} \in \mathcal{O}_{B Z}$ with mass dimension $d_{B Z}$, and $O_{S M} \in \mathcal{O}_{S M}$ with mass dimension $d_{S M}$. Low energy effects of the scale invariant $\mathcal{O}_{B Z}$ fields imply a dimensional transmutation. Thus, after the dimensional transmutation Eq.(2) is given as

*Electronic address: oozansoy@physics.wisc.edu 


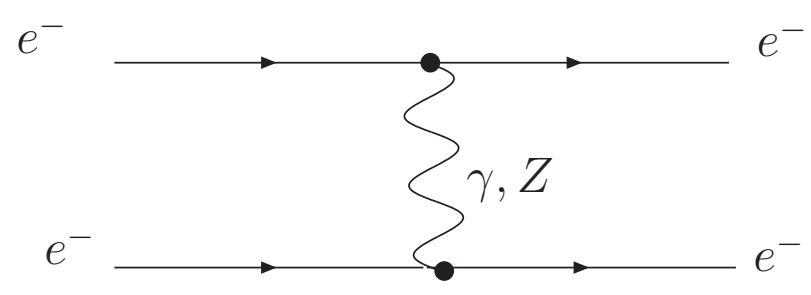

FIG. 1: The SM contribution to the scattering amplitude for $e^{-} e^{-} \rightarrow e^{-} e^{-}$scattering.

$$
\frac{C_{\mathcal{U}} \Lambda_{\mathcal{U}}^{d_{B Z}-d}}{\mathcal{M}_{\mathcal{U}}^{k}} O_{\mathcal{U}} O_{S M}
$$

where $d$ is the scaling mass dimension of the unparticle operator $O_{\mathcal{U}}$ (in the Refs. [14, 16], $d=d_{\mathcal{U}}$ ), and the constant $C_{\mathcal{U}}$ is a coefficient function.

Using the calculation techniques of the standard effective field theory one can predict possible implications of the unparticles on the particle physics phenomenology. Interactions between the unparticles and the SM fields have been listed by Ref. [17]. So far, many implications of the unparticles have been studied by several works, for example, Refs. [14, 16, 17, 18, 19, 20, 21, 22, 23, 24, 25, 26, 27, 28, 29, 30, 31, 32, 33, 34, 35, 36].

In this work, we consider the fixed target $e^{-} e^{-}$collider experiment E158 at SLAC, to search for the unparticle physics effects.

\section{PARITY VIOLATING ASYMMETRY FOR MÖLLER SCATTERING}

The tree level prediction of the SM for the parity-violating asymmetry $A_{P V}$ for low energy Möller scattering, Figure 1, is due to the interference between electromagnetic and weak neutral current amplitudes and is given by, Ref. [2],

$$
A_{P V}=-\frac{G_{F} Q^{2}}{\sqrt{2} \pi \alpha(Q)} \frac{(1-y)}{\left[1+y^{4}+(1-y)^{4}\right]}\left[1-4 \sin ^{2} \theta_{W}\right]
$$

where $G_{F}$, and $\alpha(Q)$ are the Fermi, and the fine structure constants, Ref. [37], respectively, and the momentum transfer is $Q^{2}=-q^{2}=-s y$ for $y=\frac{1}{2}(1-\cos \theta)$, and the mandelstam parameter $s$.

For the fixed target experiments, the parity-violating asymmetry is very small due to the smallness of the factor $G_{F} Q^{2}$. For the SLAC E158 experiment at a beam energy $\approx 50 \mathrm{GeV}$ and a center of mass scattering angle $90^{\circ}$, the SM tree level prediction for this asymmetry is $A_{P V}^{\text {tree }} \approx 320 \times 10^{-9}$. However, the electroweak radiative corrections, Refs. [3, 7, 8], and the experimental precision imply about $50 \%$ reduction for the measured asymmetry. The largest radiative corrections to $A_{P V}$ at low energies come from the $W W$ box diagrams, and the photonic vertex, and the box diagrams, and the $\gamma Z$ mixing and the anapole moment, Ref. [6].

Following the conventions of the Ref. [3], one can rewrite the parity-violating asymmetry with one-loop radiative corrections as

$$
A_{P V}=\frac{\rho G_{F} Q^{2}}{\sqrt{2} \pi \alpha} \frac{1-y}{1+y^{4}+(1-y)^{4}} \mathcal{F}_{Q E D} Q_{W}^{S M(e f f)}
$$

where $\rho$ is the low-energy ratio of the weak neutral and charge current couplings, $\mathcal{F}_{Q E D}=1.01 \pm 0.01$ is a QED radiation factor that includes kinematically weighted hard initial and final state radiation effects and y-dependent contributions from the $\gamma \gamma$ and $\gamma Z$ box and vertex diagrams, [13], and the SM effective weak charge is defined as

$$
Q_{W}^{S M(e f f)}=\left\{1-4 \kappa(0) s_{W}^{2}+\frac{\alpha\left(M_{Z}\right)}{4 \pi s_{W}^{2}}-\frac{3 \alpha\left(M_{Z}\right)}{32 \pi s_{W}^{2} c_{W}^{2}}\left(1-4 s_{W}^{2}\right)\left[1+\left(1-4 s_{W}^{2}\right)^{2}\right]+F_{1}\left(y, Q^{2}\right)+F_{2}\left(y, Q^{2}\right)\right\}
$$




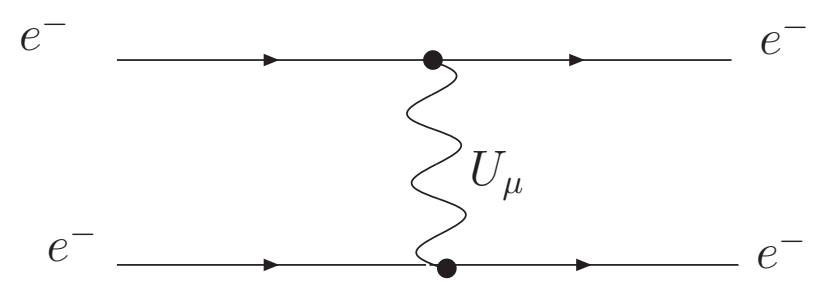

FIG. 2: The contribution to the scattering amplitude for $e^{-} e^{-} \rightarrow e^{-} e^{-}$scattering from the exchange of the vector unparticle.

where $s_{W}=\sin \theta_{W}\left(M_{Z}\right) \overline{M S}, \quad c_{W}=\cos \theta_{W}\left(M_{Z}\right) \overline{M S}$

We consider the following interactions between the Standard Model leptons and the vector unparticles [16, 17]

$$
\frac{1}{\Lambda_{U}^{d-1}} \bar{e} \gamma_{\mu}\left(\lambda_{V}-\lambda_{A} \gamma_{5}\right) e O_{\mathcal{U}}^{\mu}+\text { h.c. }
$$

where $\Lambda_{U}, \lambda_{V}$, and $\lambda_{A}$ are the unparticle energy scale, the vector, and the axial vector unparticle couplings, respectively.

Propagator for the vector unparticles has been given by

$$
\left[\Delta_{F}^{V}\left(q^{2}\right)\right]_{\mu \nu}=\frac{A_{d}}{2 \sin (d \pi)}\left[-q^{2}\right]^{d-2}\left[-g_{\mu \nu}+a \frac{q_{\mu} q_{\nu}}{q^{2}}\right]
$$

where $a=1$ corresponds to the Georgi original proposal, Ref. [14], 17], and $a=2 \frac{d-2}{d-1}$ for the conformal symmetry argument, Ref. [36], (in our calculations we assume $a=1$ ) $q$ is the momentum of the unparticle and

$$
A_{d}=\frac{16 \pi^{5 / 2}}{(2 \pi)^{2 d}} \frac{\Gamma(d+1 / 2)}{\Gamma(d-1) \Gamma(2 d)} .
$$

Therefore, the contribution to the transition amplitude of $e^{-} e^{-} \rightarrow e^{-} e^{-}$scattering from the exchange of the vector unparticle, Figure 2, takes the form

$$
\begin{aligned}
\mathcal{M}_{\mathcal{U}}=\frac{1}{\Lambda_{U}^{2 d-2}}\left[\frac{A_{d}}{2 \sin (d \pi)}\right]\{\quad & {[-t]^{d-2}\left[\bar{e}\left(p_{3}\right) \gamma_{\mu}\left(\lambda_{V}-\lambda_{A} \gamma_{5}\right) e\left(p_{1}\right)\right]\left[\bar{e}\left(p_{4}\right) \gamma^{\mu}\left(\lambda_{V}-\lambda_{A} \gamma_{5}\right) e\left(p_{2}\right)\right] } \\
& \left.-[-u]^{d-2}\left[\bar{e}\left(p_{4}\right) \gamma_{\mu}\left(\lambda_{V}-\lambda_{A} \gamma_{5}\right) e\left(p_{1}\right)\right]\left[\bar{e}\left(p_{3}\right) \gamma_{\mu}\left(\lambda_{V}-\lambda_{A} \gamma_{5}\right) e\left(p_{2}\right)\right]\right\}
\end{aligned}
$$

where $t, u$ are the mandelstam parameters.

Considering the effects of unparticles the parity-violating asymmetry can be written in the following form

$$
A_{P V}=-\frac{G_{F}}{\sqrt{2} \pi \alpha} \frac{s(1-y)}{1+y^{4}+(1-y)^{4}}\left[Q_{W}^{S M(e f f)}-\Delta Q_{W}^{U}\right]
$$

where the unparticle contribution is

$$
\Delta Q_{W}^{U}=\left\{\frac{1}{2 \sqrt{2} G_{F}}\left(\frac{A_{d}}{2 \sin (d \pi)}\right) \frac{\lambda_{A V}}{\Lambda_{U}^{2 d-2}}\left[[-t]^{d-2}+[-u]^{d-2}\right]\right\}
$$

where for the sake of brevity we use $\lambda_{A V} \equiv \lambda_{V} \lambda_{A}$.

In Figure 3, we depict the unparticle effect on the parity-violation asymmetry $A_{P V}$ with respect to the unparticle coupling $\lambda_{A V}$ for $d=1.1$ and $\Lambda=1000 \mathrm{GeV}$. From the figure, one can see that for $d=1.1$ unparticle effects are huge even the coupling $\lambda_{A V}$ is comparingly too small. This behavior is similar for $d<1.3$.

In Figure 4, and Figure 5 we depict $\Delta Q_{W}^{U} / Q_{W}$ with respect to the unparticle coupling $\lambda_{A V}$, and the scaling parameter $d$, respectively. In Figure 4 we assume $\Lambda=1000 \mathrm{GeV}$ for different values of the scaling parameter $d$. In Figure 5 we assume $\lambda_{A V}=10^{-6}$ for two different unparticle energy scale $\Lambda_{U}$. According to those figures, it is clearly seen that the unparticle effects for $d<1.3$ are very significant for the given configurations of the $\left(\lambda_{A V}, \Lambda\right)$. 


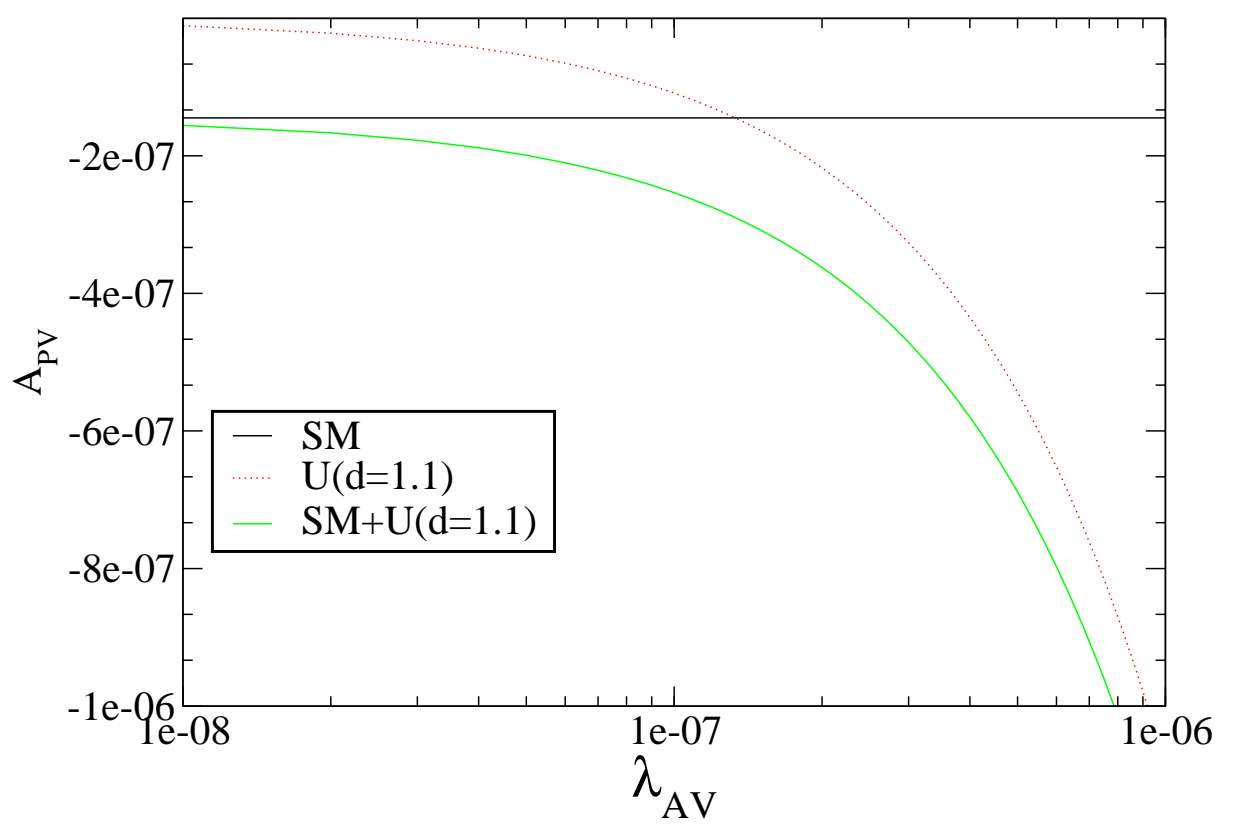

FIG. 3: $A_{P V}$ with respect to scaling parameter $\lambda_{A V}$.

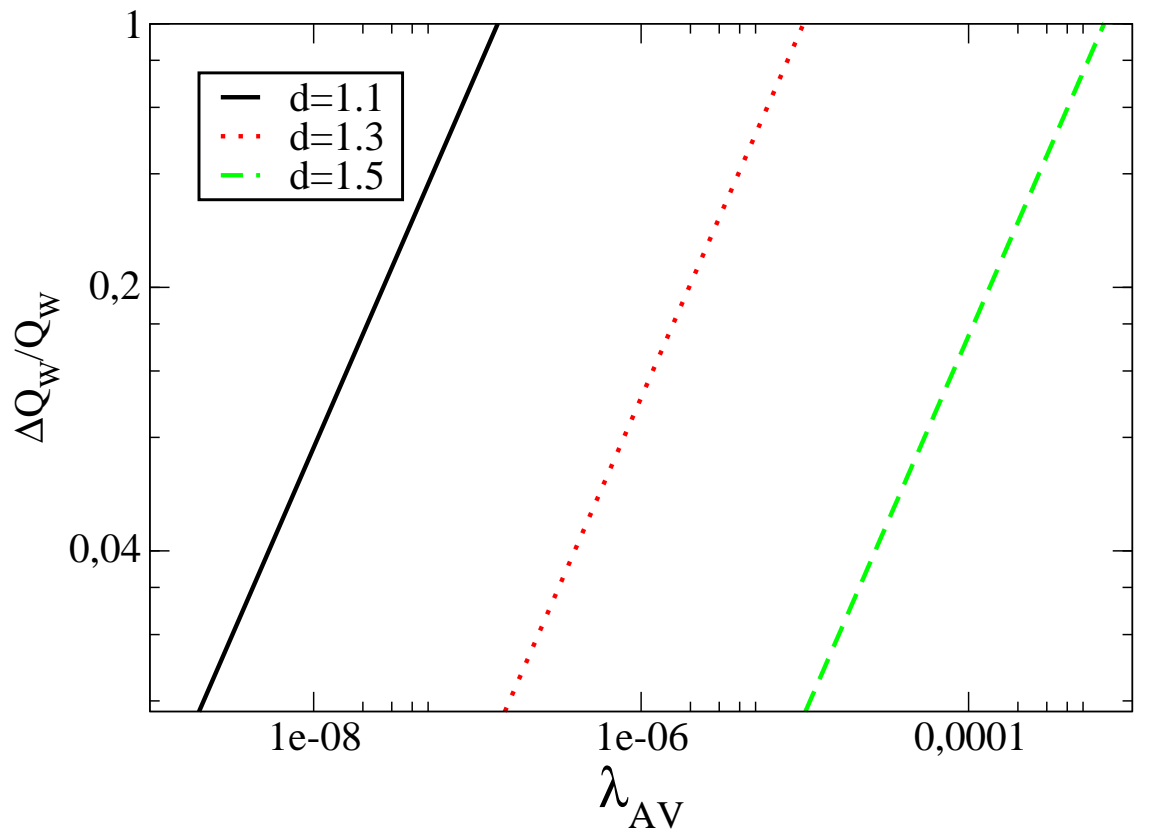

FIG. 4: $\Delta Q_{W}^{U}$ with respect to the unparticle coupling $\lambda_{A V}$ for $\Lambda=1000 \mathrm{GeV}$.

\section{RESULTS AND DISCUSSIONS}

According to the latest report given by the E158 Collaboration, Ref. [4], the combined result for the parity-violating asymmetry is 


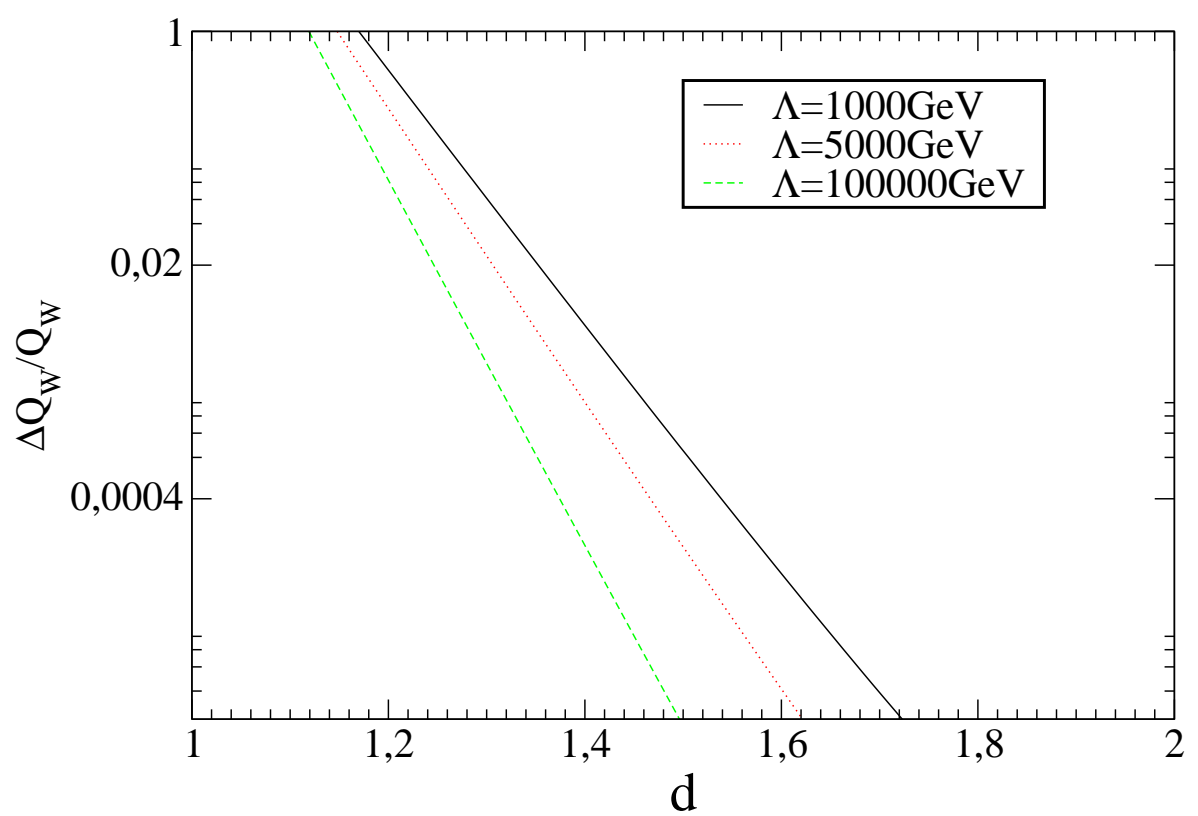

FIG. 5: $\Delta Q_{W}^{U}$ with respect to scaling parameter $d$.

TABLE I: Upper limits on the $\lambda_{A V}$ for various values of the scaling parameter $d$. Here, we assume $\Lambda_{U}=1000 \mathrm{GeV}$.

\begin{tabular}{rr}
\hline \hline $\mathrm{d}$ & $\lambda_{A V}$ \\
\hline $\mathrm{d}=1.1$ & $1.3 \times 10^{-8}$ \\
$\mathrm{~d}=1.3$ & $1.0 \times 10^{-6}$ \\
$\mathrm{~d}=1.5$ & $6.7 \times 10^{-4}$ \\
$\mathrm{~d}=1.7$ & $3.8 \times 10^{-3}$ \\
$\mathrm{~d}=1.9$ & $1.2 \times 10^{-1}$ \\
\hline \hline
\end{tabular}

$$
A_{P V}^{E X P}=-131 \pm 14(\text { stat }) \pm 10(\text { syst }) p p b
$$

where the average values of the kinematical variables are $Q^{2}=0.026 \mathrm{GeV}^{2}$, and $y=Q^{2} / s \approx 0.6$. Using this experimental result, for fixed values of the scaling dimension $d$, and assuming $\Lambda_{U}=1000 \mathrm{GeV}$, we extract the upper limits on the unparticle coupling $\lambda_{A V}$. In the calculations, we use the standard chi-square analysis for the following $\chi^{2}$ function

$$
\chi^{2}=\left[\frac{A_{P V}^{E X P}-A_{P V}^{S M+\mathcal{U}}\left(\lambda_{A V}\right)}{\delta}\right]^{2}
$$

where $\delta=\sqrt{\delta_{\text {syst }}^{2}+\delta_{\text {stat }}^{2}}$ For the one sided chi-square analysis, we assume $\chi^{2} \geq 2.7$ which corresponds to the $\% 95$ C.L. limits. Our results are given in the Table 1 .

Since unparticle contribution to the parity violating asymmetry is proportional $\lambda_{A V} / \Lambda_{U}^{2 d-2}$, using the above limits for $\lambda_{A V}$ one can plot the parameter space of $\lambda_{A V}$ versus $\Lambda_{U}$, Figure 6. In the figure, right hand side of each curve is ruled out according to the $95 \%$ C.L. analysis for corresponding scaling dimension $d$.

For a comparision the limits on the vector or the axial-vector unparticles from the literature are summarized in the Table II for the lower limits on the unparticle energy scale $\Lambda_{U}$ couplings $\lambda_{V, A}=1$ are assumed; for the upper limits on the couplings $\Lambda_{U}=1 \mathrm{TeV}$ is assumed. One can easily see that our results are very stringent and comparable the most stringent limits excisting in the literature. 


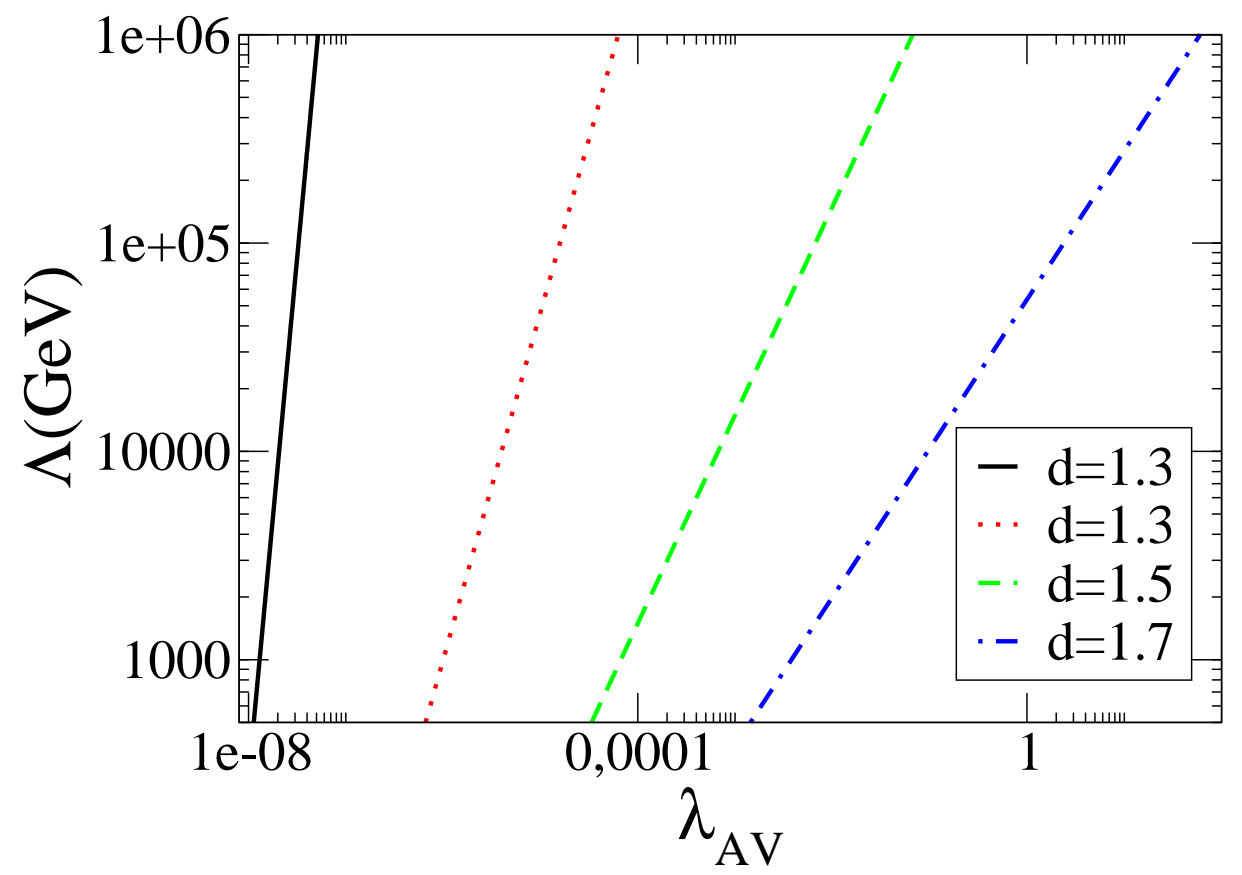

FIG. 6: Upper limits on the scalar unparticle coupling $\lambda_{0}$ depending on $\Lambda_{\mathcal{U}}$

As a conclusion, we study the unparticle effects on the parity violating asymmetry $A_{P V}$ in the low energy electronelectron scattering. We show that the parity-violating asymmetry $A_{P V}$ measurments, which are complementary to the high energy collider experiments to seek for the new physics effects, give very stringent limits on unparticles, especially for the values $d<1.3$.

We would like to remark that the recent proposal on the possibility to perform a new measurement at the Jefferson laboratory can potentialy achieve a factor of 5 improvement over the result of the E158 measurement, Ref. [11]. Therefore, such an improvement would give better understanding of the new physics effects, and can be used to put more stringent limits on the new physics scenarios, such as the unparticle physics.

\section{ACKNOWLEDGMENTS}

It is a pleasure to thank M. Ramsey-Musolf, F. Petriello, K. Kumar, and B. Balantekin for helpful conversations and discussions on this work. I also would like to thank to the members of the Nuclear Theory Group of University of Wisconsin for their hospitality. 
TABLE II: Limits on unparticles from the literature

\begin{tabular}{|c|c|c|c|}
\hline Experiment & limits for various $d$ values & & \\
\hline LEP ([18]) & $d=1.1$ & $d=1.5$ & $d=1.9$ \\
\hline$e \mu$ & $\Lambda_{U}(\mathrm{TeV})>9.1 \times 10^{14}$ & $\Lambda_{U}(T e V)>61$ & $\Lambda_{U}(T e V)>3.7$ \\
\hline$e \tau$ & $\Lambda_{U}(T e V)>9.7 \times 10^{12}$ & $\Lambda_{U}(T e V)>25$ & $\Lambda_{U}(T e V)>2.2$ \\
\hline$e q$ & $\Lambda_{U}(T e V)>2.8 \times 10^{14}$ & $\Lambda_{U}(T e V)>52$ & $\Lambda_{U}(T e V)>3.5$ \\
\hline$e b$ & $\Lambda_{U}(T e V)>1.9 \times 10^{11}$ & $\Lambda_{U}(T e V)>4.5$ & $\Lambda_{U}(\mathrm{TeV})>0.45$ \\
\hline$e^{-} e^{+} \rightarrow \gamma X([17])$ & $d=1.4$ & $d=1.6$ & $d=1.8$ \\
\hline & $\Lambda_{U}(T e V)>660$ & $\Lambda_{U}(T e V)>23$ & $\Lambda_{U}(T e V)>4$ \\
\hline Atomic parity violation ([17]) through eedd & $d=1.4$ & $d=1.5$ & $d=1.9$ \\
\hline & $\Lambda_{U}(T e V)>100$ & $\Lambda_{U}(T e V)>30$ & $\Lambda_{U}(T e V)>2$ \\
\hline Atomic parity violation ([17]) through eeuu & $d=1.4$ & $d=1.5$ & $d=1.9$ \\
\hline & $\Lambda_{U}(T e V)>100$ & $\Lambda_{U}(\mathrm{TeV})>25$ & $\Lambda_{U}(T e V)>1$ \\
\hline Atomic parity violation ([23]) for $\lambda_{A}^{e}=1, \lambda_{V}^{d, u}=1$ & $d=1.1$ & $d=1.5$ & $d=1.8$ \\
\hline & $\Lambda_{U}(\mathrm{TeV})>6$ & $\Lambda_{U}(T e V)>2$ & $\Lambda_{U}(T e V)>1$ \\
\hline$(g-2)_{e}([21],[27])$ & & $\mathrm{d}=1.5$ & \\
\hline & & $\Lambda_{V}(T e V)>37$ & \\
\hline$(g-2)_{e}([21],[27])$ & & $\mathrm{d}=1.5$ & \\
\hline & & $\Lambda_{A}(T e V)>146$ & \\
\hline$(g-2)_{\mu}([27])$ & $\mathrm{d}=1.5$ & $\mathrm{~d}=1.6$ & \\
\hline & $\Lambda_{V}(\mathrm{TeV})>1000$ & $\Lambda_{V}(T e V)>10$ & \\
\hline$(g-2)_{\mu}([27])$ & $\mathrm{d}=1.5$ & $\mathrm{~d}=1.6$ & \\
\hline & $\Lambda_{A}(T e V)>100$ & $\Lambda_{A}(T e V)>1$ & \\
\hline Invisible positronium decays ([21]) & & $\mathrm{d}=1.5$ & \\
\hline & & $\Lambda_{V}(\mathrm{TeV})>4.3 \times 10^{5}$ & \\
\hline Invisible positronium decays ([21]) & & $\mathrm{d}=1.5$ & \\
\hline & & $\Lambda_{A}(T e V)>5.1 \times 10^{2}$ & \\
\hline Atomic PV through $\mathrm{Ba}$, and $\mathrm{Yb}$ isotope chains $([24])$ & $d=1.1$ & $d=1.5$ & $d=1.9$ \\
\hline & $\lambda_{A V}<10^{-3}$ & $\lambda_{A V}<2 \times 10^{-2}$ & $\lambda_{A V}<1.1 \times 10^{-1}$ \\
\hline Low energy $\nu e$ scattering $([25])$ & $\mathrm{d}=1.1$ & $\mathrm{~d}=1.5$ & $\mathrm{~d}=1.9$ \\
\hline & $\lambda_{V}<6.2 \times 10^{-6}$ & $\lambda_{1}<9.1 \times 10^{-3}$ & $\lambda_{1}<8.1$ \\
\hline Invisible decays of $\mathrm{Z}([26])$ & & $\mathrm{d}=1.3$ & $\mathrm{~d}=1.5$ \\
\hline & & $\lambda_{V}<0.049$ & $\lambda_{V}<0.1$ \\
\hline & & $\Lambda_{V}(T e V)>10^{4}$ & \\
\hline
\end{tabular}

[1] M. J. Ramsey-Musolf, Phys. Rev. C 60, 015501 (1999) arXiv:hep-ph/9903264.

[2] E. Derman and W. J. Marciano, Annals Phys. 121 (1979) 147.

[3] A. Czarnecki and W. J. Marciano, "Electroweak radiative corrections to polarized Moller scattering Phys. Rev. D 53, 1066 (1996) arXiv:hep-ph/9507420.

[4] P. L. Anthony et al. [SLAC E158 Collaboration], Phys. Rev. Lett. 95, 081601 (2005) arXiv:hep-ex/0504049.

[5] P. L. Anthony et al. [SLAC E158 Collaboration], Phys. Rev. Lett. 92, 181602 (2004) arXiv:hep-ex/0312035.

[6] A. Czarnecki and W. J. Marciano, Int. J. Mod. Phys. A 15, 2365 (2000) arXiv:hep-ph/0003049.

[7] A. Kurylov, M. J. Ramsey-Musolf and S. Su, Phys. Rev. D 68, 035008 (2003) arXiv:hep-ph/0303026.

[8] J. Erler and M. J. Ramsey-Musolf, Phys. Rev. D 72, 073003 (2005) arXiv:hep-ph/0409169.

[9] K. S. Kumar, Eur. Phys. J. A 24S2, 191 (2005).

[10] K. S. Kumar, In the Proceedings of 1996 DPF / DPB Summer Study on New Directions for High-Energy Physics (Snowmass 96), Snowmass, Colorado, 25 Jun - 12 Jul 1996, pp NEW168.

[11] K. S. Kumar [E158 Collaboration], AIP Conf. Proc. 792, 353 (2005).

[12] M. J. Musolf etal. Phys. Rept. 239(1994)1.

[13] Zykunov et al., hep-ph/0507287

[14] H. Georgi, Phys. Rev. Lett. 98, 221601 (2007) arXiv:hep-ph/0703260.

[15] T. Banks and A. Zaks, Nucl. Phys. B 196, 189 (1982).

[16] H. Georgi, Phys. Lett. B 650, 275 (2007) arXiv:0704.2457 [hep-ph]].

[17] K. Cheung, W. Y. Keung and T. C. Yuan, Phys. Rev. Lett. 99, 051803 (2007) arXiv:0704.2588 [hep-ph]; Phys. Rev. D 76, 055003 (2007) arXiv:0706.3155 [hep-ph]]. S. L. Chen, X. G. He and H. C. Tsai, arXiv:0707.0187 [hep-ph].

[18] M. Bander, J. L. Feng, A. Rajaraman and Y. Shirman, Phys. Rev. D 76, 115002 (2007) arXiv:0706.2677] [hep-ph]];

[19] L. Anchordoqui and H. Goldberg, arXiv:0709.0678 [hep-ph]. 
[20] S. Hannestad, G. Raffelt and Y. Y. Y. Wong, Phys. Rev. D 76, 121701 (2007) arXiv:0708.1404 [hep-ph]];

[21] Y. Liao, Phys. Rev. D 76, 056006 (2007) arXiv:0705.0837 [hep-ph]].

[22] H. Davoudiasl, Phys. Rev. Lett. 99, 141301 (2007) arXiv:0705.3636 [hep-ph]];

[23] G. Bhattacharyya, D. Choudhury and D. K. Ghosh, Phys. Lett. B 655, 261 (2007) arXiv:0708.2835 [hep-ph]];

[24] G. J. Ding and M. L. Yan, Phys. Rev. D 76, 075005 (2007) arXiv:0705.0794 [hep-ph]];

[25] A. B. Balantekin and K. O. Ozansoy, arXiv:0710.0028 [hep-ph].

[26] S. L. Chen, X. G. He and H. C. Tsai, JHEP 0711, 010 (2007) arXiv:0707.0187 [hep-ph]];

[27] T. G. Rizzo, JHEP 0710, 044 (2007) arXiv:0706.3025 [hep-ph]];

[28] T. M. Aliev, A. S. Cornell and N. Gaur, Phys. Lett. B 657, 77 (2007) arXiv:0705.1326 [hep-ph]].

[29] O. Cakir and K. O. Ozansoy, Eur. Phys. J. C 56, 279 (2008) arXiv:0712.3814 [hep-ph]].

[30] O. Cakir and K. O. Ozansoy, Europhys. Lett. 83, 51001 (2008) arXiv:0710.5773 [hep-ph]].

[31] P. J. Fox, A. Rajaraman and Y. Shirman, Phys. Rev. D 76, 075004 (2007) arXiv:0705.3092 [hep-ph]];

[32] E. O. Iltan, arXiv:0710.2677 [hep-ph];

[33] A. Lenz, Phys. Rev. D 76, 065006 (2007) arXiv:0707.1535 [hep-ph]];

[34] A. T. Alan and N. K. Pak, arXiv:0708.3802 [hep-ph];

[35] I. Sahin and B. Sahin, Eur. Phys. J. C 55, 325 (2008) arXiv:0711.1665 [hep-ph]].

[36] B. Grinstein, K. Intriligator and I. Z. Rothstein, Phys. Lett. B 662, 367 (2008) arXiv:0801.1140 [hep-ph]].

[37] W. M. Yao et al. [Particle Data Group], J. Phys. G 33, 1 (2006). 